\title{
A duality perspective on Loewner rational interpolation and state-space modelling of vector-exponential trajectories
}

\author{
P. Rapisarda and A.C. Antoulas
}

\begin{abstract}
We explore some connections between the Loewner approach to interpolation and realization, and that based on bilinear differential forms arising in the behavioral framework. We show that a crucial concept underlying both approaches is that of duality of trajectories, and that many known results can be interpreted in its light.
\end{abstract}

\section{INTRODUCTION}

The Loewner framework was initiated in [16], [17] in the context of tangential interpolation and partial realization problems (see also [1], [4]), and has been successfully applied also to model order reduction (see [2], [3], [14], [15]). Vector-exponential time-series modelling from a behavioral perspective has been introduced in [28], and applied to metric interpolation problems in [12], [13], [25]. Some connections between rational interpolation and behavioral modelling of vector-exponential trajectories were first explored in [6], and inform also more recent approaches (see [19], [21]) to modelling based on bilinear- and quadratic differential forms (B/QDFs in the following, see [29]).

The purpose of the present paper is to offer a novel point of view on the Loewner- and the BDF-based approach, based on the notion of duality of trajectories. We show that crucial results in both approaches can be interpreted and formulated in terms of (generalized) state-input-output representations of the primal- and the dual system. Of particular relevance is that the factorization of the Loewner matrix, that plays a crucial role in obtaining state models from vector-exponential data, can be given a trajectory-based interpretation based on duality.

The paper is organized as follows. In section II we formulate the problem of (bi-directional) rational interpolation, and we introduce the concept of Loewner matrix. In section III we use input-state-output representations and duality to show how state trajectories corresponding to the inputoutput data can be constructed from the factorization of the Loewner matrix. In section IV we extend such results to the case of systems represented by generalized input-state-output equations. In section $\mathrm{V}$ we consider the higher-order case, summarizing some of the results already presented in a more thorough form in [18].

P. Rapisarda is with the Vision, Learning and Control Group, School of Electronics and Computer Science, University of Southampton, SO17 1BJ Southampton, UK pr3eecs.soton.ac.uk

A.C. Antoulas is with the Department of Electrical and Computer Engineering, Rice University, Houston, TX 77005, USA, aca@rice.edu, and the School of Engineering and Science, Jacobs University, Bremen, Germany
Finally, section VI contains the conclusions and lists some directions of current and future research.

\section{NOTATION}

The space of $n$ dimensional real (complex) vectors is denoted by $\mathbb{R}^{n}$ (respectively $\mathbb{C}^{n}$ ), and that of $m \times n$ real matrices by $\mathbb{R}^{m \times n}$. $\mathbb{R}^{\bullet \times m}$ denotes the space of real matrices with $m$ columns and an unspecified finite number of rows. Given matrices $A, B \in \mathbb{R}^{\bullet \times m}, \operatorname{col}(A, B)$ denotes the matrix obtained by stacking $A$ over $B$.

The ring of polynomials with real coefficients in the indeterminate $\xi$ is denoted by $\mathbb{R}[\xi]$; the ring of two-variable polynomials with real coefficients in the indeterminates $\zeta$ and $\eta$ is denoted by $\mathbb{R}[\zeta, \eta] . \mathbb{R}^{r \times q}[\xi]$ denotes the set of all $r \times q$ matrices with entries in $\xi$, and $\mathbb{R}^{n \times m}[\zeta, \eta]$ that of $n \times m$ polynomial matrices in $\zeta$ and $\eta$. The set of rational $m \times n$ matrices in the indeterminate $\xi$ is denoted by $\mathbb{R}^{m \times n}(\xi)$.

The set of infinitely differentiable functions from $\mathbb{R}$ to $\mathbb{R}^{q}$ is denoted by $\mathfrak{C}^{\infty}\left(\mathbb{R}, \mathbb{R}^{q}\right)$.

\section{PROBlem FORMULATION}

Define the left and right interpolation data as the triples in $\mathbb{C} \times \mathbb{C}^{p} \times \mathbb{C}^{m}$ and $\mathbb{C} \times \mathbb{C}^{m} \times \mathbb{C}^{p}$, respectively:

$$
\begin{array}{ll}
\left\{\left(\mu_{i}, \ell_{i}^{*}, v_{i}^{*}\right)\right\}_{i=1, \ldots, k_{1}}, \quad \mu_{i} \in \mathbb{C}, \ell_{i}^{*} \in \mathbb{C}^{1 \times p}, v_{i}^{*} \in \mathbb{C}^{1 \times m} \\
\left\{\left(\lambda_{i}, r_{i}, w_{i}\right)\right\}_{i=1, \ldots, k_{2}}, \quad \lambda_{i} \in \mathbb{C}, r_{i} \in \mathbb{C}^{m}, w_{i} \in \mathbb{C}^{p} .(1)
\end{array}
$$

In the rest of this paper we will assume for simplicity of exposition that the $\mu_{i} s$ and $\lambda_{i} s$ are distinct; the general case follows with straightforward modifications of the statements and the arguments. We will also assume that $\left\{\mu_{i}\right\}_{i=1, \ldots, k_{1}} \cap$ $\left\{\lambda_{j}\right\}_{j=1, \ldots, k_{2}}=\emptyset$.

Let $H \in \mathbb{R}^{p \times m}(\xi)$ be a proper rational matrix. $H$ satisfies the interpolation constraints if

$$
\begin{aligned}
\ell_{i}^{*} H\left(\mu_{i}\right) & =v_{i}^{*}, i=1, \ldots, k_{1} \\
H\left(\lambda_{i}\right) r_{i} & =w_{i}, i=1, \ldots, k_{2} .
\end{aligned}
$$

If only the first, respectively second, set of constraints (2) is satisfied, we call $H$ a left-, respectively right interpolant. The problem we consider in this paper is that of deriving a right/left interpolant $H \in \mathbb{R}^{p \times m}(\xi)$ from data (1). The following constant matrices play a crucial role in the solution of this problem. The Loewner matrix associated with the interpolation data (1) is defined by

$$
\mathbb{L}:=\left[\frac{v_{i}^{*} r_{j}-\ell_{i}^{*} w_{j}}{\mu_{i}-\lambda_{j}}\right]_{i=1, \ldots, k_{1} ; j=1, \ldots, k_{2}} .
$$

The shifted Loewner matrix is defined by

$$
\sigma \mathbb{L}:=\left[\frac{\mu_{i} v_{i}^{*} r_{j}-\lambda_{j} \ell_{i}^{*} w_{j}}{\mu_{i}-\lambda_{j}}\right]_{i=1, \ldots, k_{1} ; j=1, \ldots, k_{2}} .
$$




\section{INPUT-STATE-OUTPUT EQUATIONS FROM DATA}

Consider a system described in minimal input-state-output form by the equations

$$
\begin{aligned}
\frac{d}{d t} x & =A x+B u \\
y & =C x+D u ;
\end{aligned}
$$

its dual is minimally represented by the equations

$$
\begin{aligned}
\frac{d}{d t} x^{\prime} & =-A^{\top} x^{\prime}+C^{\top} u^{\prime} \\
y^{\prime} & =B^{\top} x^{\prime}-D^{\top} u^{\prime} .
\end{aligned}
$$

We now state an important relation between the input-output and the state trajectories of (5) and (6).

Proposition 1: Let $\operatorname{col}(u, y, x)$ and $\operatorname{col}\left(u^{\prime}, y^{\prime}, x^{\prime}\right)$ satisfy (5) and (6), respectively. Then

$$
u^{\prime *} y+y^{\prime *} u=\frac{d}{d t}\left(x^{*} x\right)
$$

Proof: The claim follows from the chain of equalities:

$$
\begin{aligned}
u^{\prime *} y+y^{\prime *} u & =u^{\prime *}(C x+D u)+\left(B^{\top} x^{\prime}-D^{\top} u^{\prime}\right)^{*} u \\
& =\left(u^{\prime *} C\right) x+x^{\prime *}(B u) \\
& =\left(\frac{d}{d t} x^{\prime}+A^{\top} x^{\prime}\right)^{*} x+x^{*}\left(\frac{d}{d t} x-A x\right) \\
& =\frac{d}{d t}\left(x^{\prime *} x\right) .
\end{aligned}
$$

Now consider the case when $\operatorname{col}(u, y)$ and $\operatorname{col}\left(u^{\prime}, y^{\prime}\right)$ are vector-exponential, i.e. there exist constant vectors $\bar{w}:=$ $\operatorname{col}(\bar{u}, \bar{y}) \in \mathbb{C}^{m+p}$ and $\overline{w^{\prime}}:=\operatorname{col}\left(\overline{u^{\prime}}, \overline{y^{\prime}}\right) \in \mathbb{C}^{p+m}$ and $\mu, \lambda \in \mathbb{C}$ such that

$$
\operatorname{col}(u, y)(\cdot)=\bar{w} e^{\mu \cdot} \text { and } \operatorname{col}\left(u^{\prime}, y^{\prime}\right)(\cdot)=\overline{w^{\prime}} e^{\lambda \cdot} .
$$

It is straightforward to check that also the corresponding state trajectories are vector-exponential, i.e. there exist $\bar{x}, \overline{x^{\prime}} \in \mathbb{C}^{n}$ such that

$$
x(\cdot)=\bar{x} e^{\mu \cdot} \text { and } x^{\prime}(\cdot)=\overline{x^{\prime}} e^{\lambda \cdot} .
$$

It follows in a straightforward manner from the result of Prop. 1 that for such vector-exponential trajectories the following relation holds:

$$
{\overline{w^{\prime}}}^{*} \bar{w}=\left(\lambda^{*}+\mu\right){\overline{x^{\prime}}}^{*} \bar{x} .
$$

Consequently, given a pair of finite families of input-output vector-exponential trajectories

$$
\left\{\overline{w^{\prime}}{ }_{i} e^{\lambda_{i} \cdot}\right\}_{i=1, \ldots, N} \text { and }\left\{\bar{w}_{j} e^{\mu_{j} \cdot}\right\}_{j=1, \ldots, N},
$$

under the assumption $\lambda_{i}^{*} \neq-\mu_{j}, i, j=1, \ldots, N$, equation (8) implies that

$$
\left[\frac{{\overline{w^{\prime}}}_{i}^{*} \bar{w}_{j}}{\lambda_{i}^{*}+\mu_{j}}\right]_{i, j=1, \ldots, N}=\left[{\overline{x^{\prime}}}_{i}^{*} \bar{x}_{j}\right]_{i, j=1, \ldots, N} .
$$

Given a matrix $S \in \mathbb{R}^{k_{1} \times k_{2}}$, a rank-revealing factorization of $S$ is any factorization $S=U_{1} U_{2}$ with $U \in \mathbb{R}^{k_{1} \times n}$, $U_{2} \in \mathbb{R}^{n \times k_{2}}$ of full rank $n=\operatorname{rank} S$. Such a factorization can be computed in a straightforward way for example from a singular value decomposition of $S$. Now observe that the left-hand side of equation (9) is the analogous of the Loewner matrix $\mathbb{L}$ defined in (3); moreover, the relation (9) between external- and internal (state) data also implies that any rank-revealing factorization of the left-hand side yields (the directions of) minimal state trajectories associated with the external data.

The following result shows that under mild conditions, from a factorization of the Loewner matrix one can obtain a number of linearly independent state trajectories equal to the McMillan degree $n$ of the system. This result is analogous to Lemma 2.1 in [16].

Proposition 2: Denote by $n$ the McMillan degree of the state-representations (5) and (6), and with $N$ the number of input-output data trajectories. Assume that $N \geq n$, that $\lambda_{i}^{*} \neq-\mu_{j}, i, j=1, \ldots, N$, and that $\lambda_{i} \neq \lambda_{j}$ and $\mu_{i} \neq \mu_{j}$ for $i \neq j$. Then

$$
\operatorname{rank}\left[\frac{{\overline{w^{\prime}}}_{i}^{*} \bar{w}_{j}}{\lambda_{i}^{*}+\mu_{j}}\right]_{i, j=1, \ldots, N}=n .
$$

Proof: Using (9) conclude that $\left[\frac{{\overline{w^{\prime}}}_{i}^{*} \bar{w}_{j}}{\lambda_{i}^{*}+\mu_{j}}\right]_{i, j=1, \ldots, N}=$ $S^{*} P$, where $S$ and $P$ are defined by

$$
\begin{aligned}
S & :=\left[\begin{array}{lll}
\bar{x}_{1}^{\prime} & \ldots & \bar{x}_{N}^{\prime}
\end{array}\right] \in \mathbb{C}^{n \times N} \\
P & :=\left[\begin{array}{lll}
\bar{x}_{1} & \ldots & \bar{x}_{N}
\end{array}\right] \in \mathbb{C}^{n \times N} .
\end{aligned}
$$

We now prove that under the assumption that the $\lambda_{i} \mathrm{~s}$ are distinct, the matrix $P$ has full row rank $n$; a similar argument yields the same property for $S$.

Assume by contradiction that $\operatorname{rank}(P)=r<n$; then without loss of generality we can assume that there exist $\alpha_{i} \in \mathbb{C}, i=1, \ldots, n$, not all zero, such that $P^{\prime} \operatorname{col}\left(\alpha_{i}\right)_{i=1, \ldots, n}=0$, where $P^{\prime}$ is the submatrix of $P$ consisting of its first $n$ columns. Consider the state trajectory $\hat{x}(\cdot):=\sum_{i=1}^{n} \alpha_{i} \bar{x}_{i} e^{\lambda_{i} \cdot}$, corresponding to the input-output trajectory $\operatorname{col}(\hat{u}, \hat{y})(\cdot):=\sum_{i=1}^{n} \alpha_{i} \operatorname{col}\left(\bar{u}_{i}, \bar{y}_{i}\right) e^{\lambda_{i}} \cdot$. The external behavior spanned by the trajectories $\operatorname{col}\left(\bar{u}_{i}, \bar{y}_{i}\right) e^{\lambda_{i} \cdot}$ is autonomous (see [27]), i.e. without inputs. Moreover $x$ is a state variable for such behavior. Now observe that $\hat{x}(0)=0$; it follows that $\operatorname{col}(\hat{u}, \hat{y})$ is also zero. However this is in contradiction with the assumption that not all $\alpha_{i}$ 's are equal to zero and that the $\lambda_{i} \mathrm{~s}$ are distinct. Consequently $P$ has rank $n$.

The following result is analogous to the first formula in (12) p. 640 of [16]).

Proposition 3: Define the matrices

$$
\begin{aligned}
M & :=\operatorname{diag}\left(\mu_{i}\right)_{i=1, \ldots, N} \\
\Lambda & :=\operatorname{diag}\left(\lambda_{j}\right)_{j=1, \ldots, N} \\
L & :=\left[\begin{array}{lll}
\overline{w^{\prime}} & \ldots & \bar{w}_{N}^{\prime}
\end{array}\right] \in \mathbb{C}^{(m+p) \times N} \\
R & :=\left[\begin{array}{lll}
\bar{w}_{1} & \ldots & \bar{w}_{N}
\end{array}\right] \in \mathbb{C}^{(m+p) \times N} .
\end{aligned}
$$

The matrix $\left[\frac{{\overline{w^{\prime}}}_{i}^{*} \bar{w}_{j}}{\lambda_{i}^{*}+\mu_{j}}\right]_{i, j=1, \ldots, N}$ satisfies the Sylvester equation

$$
M\left[\frac{{\overline{w_{i}^{\prime}}}_{i}^{*} \bar{w}_{j}}{\lambda_{i}^{*}+\mu_{j}}\right]_{i, j=1, \ldots, N}+\left[\frac{{\overline{w^{\prime}}}_{i}^{*} \bar{w}_{j}}{\lambda_{i}^{*}+\mu_{j}}\right]_{i, j=1, \ldots, N} \Lambda=L^{*} R .
$$


(9).

Proof: The claim follows in a straightforward way from

Remark 1: For the case of lossless port-Hamiltonian system (see [21], [22] for the definition) and symmetric data, i.e. $\mu_{i}=\lambda_{i}, i=1, \ldots, N$, the Loewner matrix (3) coincides with the Pick matrix defined in formula (1) in [21]. The result of Prop. 2 of this paper coincides with the result of Prop. 1 of [21], and the Sylvester equation result of Prop. 3 coincides with that of Prop. 2 of [21].

Similarly, for the case of self-adjoint port-Hamiltonian systems (see [21] for the definition) and symmetric data, the Loewner matrix (3) coincides with the Pick matrix of formula (34) in [21]. Results analogous to Prop. 2 and Prop. 3 of this paper appear as Prop. 6 and Prop. 7, respectively, in [21].

Under the assumptions of Prop. 2, in order to find an inputstate-output (iso) representation (5) of an interpolant for a finite family of primal input-output data $\left\{\bar{w}_{i} e^{\mu_{i}{ }^{\cdot}}\right\}_{i=1, \ldots, N}$, one can proceed as follows. Compute a rank-revealing factorization $\left[\frac{\bar{w}_{i}^{*} \bar{w}_{j}}{\lambda_{i}^{*}+\mu_{j}}\right]_{i, j=1, \ldots, N}=S^{*} P$, and define

$$
\begin{aligned}
U & :=\left[\begin{array}{lll}
\bar{u}_{1} & \ldots & \bar{u}_{N}
\end{array}\right] \in \mathbb{C}^{m \times N} \\
Y & :=\left[\begin{array}{lll}
\bar{y}_{1} & \ldots & \bar{y}_{N}
\end{array}\right] \in \mathbb{C}^{p \times N} .
\end{aligned}
$$

The following result, whose proof is straightforward and hence omitted, characterizes input-state-output representations of right interpolants.

Proposition 4: Define $M$ as in Prop. 3, and $U$ and $Y$ by (11). A quadruple $(A, B, C, D) \in \mathbb{R}^{n \times n} \times \mathbb{R}^{n \times n} \times$ $\mathbb{R}^{n \times m} \times \mathbb{R}^{p \times n} \times \mathbb{R}^{m \times m}$ defines an iso representation of a right interpolant $H(\xi):=C\left(\xi I_{n}-A\right)^{-1} B+D$ for the primal set of data $\left(\mu_{i}, \bar{u}_{i}, \bar{y}_{i}\right)$ if and only if

$$
\left[\begin{array}{c}
P M \\
Y
\end{array}\right]=\left[\begin{array}{ll}
A & B \\
C & D
\end{array}\right]\left[\begin{array}{l}
P \\
U
\end{array}\right] \text {. }
$$

A result analogous to that of Prop. 4 holds true also for left-interpolants; we will not state it explicitly.

Remark 2: The solution of the linear system of equations (12) involves standard linear algebra computations; we will not deal with such details here.

Remark 3: In our approach we use the data to first compute state trajectories corresponding to it, and in a second stage we compute a state representation for the data and the identified state trajectories by solving linear equations in the unknown state-, input- and output matrices (see Prop. 4). Such two-stage approach is analogous to that of subspace identification (see e.g. [8]). However, our methodology does not exploit the shift-invariance of data trajectories, but rather the fact that external properties, i.e. properties at the level of external variables, in our case duality, are reflected into internal properties, i.e. at the level of state.

Remark 4: In our basis-free approach, state directions are computed from the factorization of the Loewner matrix, and determine through the equations (12) the state representation. It follows that judiciously factorizing the Loewner matrix yields special state representations, e.g. "balanced" ones, that make evident the influence of state variables on the $\mathrm{i} / \mathrm{o}$ behavior of the system. See [23] for an application to the data-driven model reduction problem.

Remark 5: For pedagogical purposes we have proceeded as if it were necessary to have available data also from the dual system; however, this is not necessary, and dual trajectories can be derived from primal ones by the following mirroring procedure (see [12], [13], [25]). The following result, which we state without proof, is crucial.

Proposition 5: Let $w(\cdot)=\operatorname{col}(\bar{u}, \bar{y}) e^{\lambda \cdot}$ be an input-output trajectory of the primal system. Let $\bar{v} \in \mathbb{C}^{m+p}$ be such that $\bar{v}^{*} \bar{w}=0$. Then $\bar{v} e^{-\lambda^{*}}$. is a trajectory of the dual system.

Thus to obtain dual vector-exponential trajectories it is sufficient to mirror primal trajectories computing a direction orthogonal to the primal one.

\section{GENERALIZED INPUT-STATE-OUTPUT EQUATIONS FROM DATA}

In this section we consider the case of a system described by the equations

$$
\begin{aligned}
E \frac{d}{d t} x & =A x+B u \\
y & =C x .
\end{aligned}
$$

We also consider its dual (note that the terminology "dual" is not uniform in the literature; on this issue see also [7], [10], [11]), represented by

$$
\begin{aligned}
E^{\top} \frac{d}{d t} x^{\prime} & =-A^{\top} x^{\prime}+C^{\top} u^{\prime} \\
y^{\prime} & =B^{\top} x^{\prime} .
\end{aligned}
$$

The following two results are crucial for computing $E$ and $A$ from factorizations of the Loewner matrices.

Proposition 6: Let $\operatorname{col}(u, y, x)$ and $\operatorname{col}\left(u^{\prime}, y^{\prime}, x^{\prime}\right)$ satisfy (13) and (14), respectively. Then

$$
\frac{d}{d t}\left(x^{\prime *} E x\right)=u^{\prime *} y+y^{\prime *} u .
$$

Proof: The claim follows from the following manipulations:

$$
\begin{aligned}
\frac{d}{d t}\left(x^{\prime *} E x\right) & =\left(-A^{\top} x^{\prime}+C^{\top} u^{\prime}\right)^{*} x+x^{\prime *}(A x+B u) \\
& =u^{\prime *} C x+x^{\prime *} B u \\
& =u^{\prime *}(C x)+\left(B^{\top} x^{\prime}\right)^{*} u \\
& =u^{\prime *} y+y^{\prime *} u .
\end{aligned}
$$

Proposition 7: Let $\operatorname{col}(x, u, y)$ and $\operatorname{col}\left(z, u^{\prime}, y^{\prime}\right)$ satisfy (13) and (14), respectively. Then

$$
\frac{d}{d t}\left(x^{\prime *} A x\right)=u^{\prime *}\left(\frac{d}{d t} y\right)-\left(\frac{d}{d t} y^{\prime *}\right) u .
$$


Proof: The claim follows from:

$$
\begin{aligned}
\frac{d}{d t}\left(x^{*} A x\right)= & \left(\frac{d}{d t} x^{\prime}\right)^{*} A x+x^{*} A\left(\frac{d}{d t} x\right) \\
= & \left(\frac{d}{d t} x^{\prime}\right)^{*}\left(E \frac{d}{d t} x-B u\right) \\
& +\left(-E^{\top} \frac{d}{d t} x^{\prime}+C^{\top} u^{\prime}\right)^{*}\left(\frac{d}{d t} x\right) \\
= & -\left(B^{\top} \frac{d}{d t} x^{\prime}\right)^{*} u+u^{\prime *}\left(C \frac{d}{d t} x\right) \\
= & \left(-\frac{d}{d t} y^{\prime *}\right) u+u^{*}\left(\frac{d}{d t} y\right) .
\end{aligned}
$$

If $\operatorname{col}(u, y)$ and $\operatorname{col}\left(u^{\prime}, y^{\prime}\right)$ are vector-exponential trajectories of the primal, respectively dual system, then the associated state trajectories are also vector-exponential. Using the same argument as that following Prop. 1, we conclude from Prop. 6 and Prop. 7 that given a pair of finite families of input-output vector-exponential trajectories

$$
\left\{\bar{w}_{i}^{\prime} e^{\lambda_{i} \cdot}\right\}_{i=1, \ldots, N} \text { and }\left\{\bar{w}_{j} e^{\mu_{j} \cdot}\right\}_{j=1, \ldots, N},
$$

and equations (15) and (16) imply that

$$
\begin{aligned}
{\left[\frac{{\overline{w^{\prime}}}_{i}^{*} \bar{w}_{j}}{\lambda_{i}^{*}+\mu_{j}}\right]_{i, j=1, \ldots, N} } & =\left[{\overline{x^{\prime}}}_{i}^{*} E \bar{x}_{j}\right]_{i, j=1, \ldots, N} \\
{\left[\frac{{\overline{u^{\prime}}}_{i}^{*} \mu_{j} \bar{y}_{j}-\lambda_{i}^{*}{\overline{y^{\prime}}}_{i}^{*} \bar{u}_{j}}{\lambda_{i}^{*}+\mu_{j}}\right]_{i, j=1, \ldots, N} } & =\left[{\overline{x^{\prime}}}_{i}^{*} A \bar{x}_{j}\right]_{i, j=1, \ldots, N}
\end{aligned}
$$

The left-hand side of the first equation in (17) is analogous to the Loewner matrix introduced in the previous section, while the left-hand side of the second equation is analogous to the shifted Loewner matrix (4).

Denote

$$
\begin{aligned}
\mathbb{L} & :=\left[\frac{{\overline{w^{\prime}}}_{i}^{*} \bar{w}_{j}}{\bar{\lambda}_{i}^{*}+\mu_{j}}\right]_{i, j=1, \ldots, N} \\
\mathbb{L}_{\sigma} & :=\left[\frac{{\overline{u^{\prime}}}_{i}^{*} \mu_{j} \bar{y}_{j}-\lambda_{i}^{*}{\overline{y^{\prime}}}_{i}^{*} \bar{u}_{j}}{\lambda_{i}^{*}+\mu_{j}}\right]_{i, j=1, \ldots, N} ;
\end{aligned}
$$

it follows from the equations (17) that there exist matrices $X^{\prime}, X \in \mathbb{C}^{n \times N}$, whose columns are the directions associated with the vector-exponential state trajectories corresponding to the input-output data, such that

$$
\begin{aligned}
{\left[\begin{array}{ll}
\mathbb{L} & \mathbb{L}_{\sigma}
\end{array}\right] } & =X^{\prime *}\left[\begin{array}{ll}
E X & A X
\end{array}\right] \\
{\left[\begin{array}{c}
\mathbb{L} \\
\mathbb{L}_{\sigma}
\end{array}\right] } & =\left[\begin{array}{l}
X^{\prime *} E \\
X^{\prime *} A
\end{array}\right] X .
\end{aligned}
$$

The equations (18) are the counterpart of those in formula (2.25) of [5], with $Y=X^{* *}, \Sigma_{\ell} \widetilde{X}^{*}=\left[\begin{array}{ll}E X & A X\end{array}\right]$ and $\tilde{Y} \Sigma_{r}=\left[\begin{array}{l}X^{\prime *} E \\ X^{\prime *} A\end{array}\right]$. A "short" SVD of the two matrices on the left-hand side of (18) yields matrices $X^{\prime *}$ and $X$ with orthonormal rows; under such assumption we recover $E$ and $A$ by projection of $\mathbb{L}$ and $\mathbb{L}_{s}$ as

$$
\begin{aligned}
E & =X^{\prime} \mathbb{L}_{X^{*}} \\
A & =X^{\prime} \mathbb{L}_{s} X^{*},
\end{aligned}
$$

respectively, see the first two formulas (22) p. 646 of [16].
From the output equation $y^{\prime}=B^{\top} x^{\prime}$ of the dual system (14) it follows that $Y^{\prime}=B^{\top} X^{\prime}$, where

$$
Y^{\prime}:=\left[\begin{array}{lll}
{\overline{y^{\prime}}}_{1} & \ldots & {\overline{y^{\prime}}}_{N}
\end{array}\right] \in \mathbb{C}^{m \times N} .
$$

Assuming that $X^{\prime}$ has been obtained via a short SVD and consequently that its rows are orthonormal, it follows that

$$
B=X^{\prime} Y^{\prime *} \text {. }
$$

This is the third equation in (2.28) p. 17 of [5]. Analogously, from the output equation $y=C x$ of the primal system (13) it follows that $Y=C X$, where

$$
Y:=\left[\begin{array}{lll}
\bar{y}_{1} & \ldots & \bar{y}_{N}
\end{array}\right] \in \mathbb{C}^{m \times N} .
$$

Consequently, assuming that $X$ has been obtained via a short SVD and consequently its rows are orthonormal,

$$
C=Y X^{*}
$$

the fourth equation in (2.28) p. 17 of [5].

\section{THE HIGHER-ORDER CASE}

We now sketch (see [18] for a thorough exposition) how to deal with the controllable higher-order case, in which the system generating the data is represented in kernel form

$$
P\left(\frac{d}{d t}\right) y=Q\left(\frac{d}{d t}\right) u,
$$

where $P \in \mathbb{R}^{p \times p}[\xi], Q \in \mathbb{R}^{p \times m}[\xi]$ are left-coprime. In such case it can be shown that the set of trajectories $\operatorname{col}(u, y)$ satisfying (19) can also be represented in image form by

$$
\operatorname{col}(u, y)=\left[\begin{array}{l}
D\left(\frac{d}{d t}\right) \\
N\left(\frac{d}{d t}\right)
\end{array}\right] \ell
$$

where $\ell$ is an auxiliary variable and $D \in \mathbb{R}^{m \times m}[\xi]$ and $N \in$ $\mathbb{R}^{p \times m}[\xi]$ are right-coprime matrices. Moreover, $P N=Q D$.

In the higher-order case, the notion of duality is defined as follows. Denote by $\mathfrak{B}$ the set of infinitely-differentiable trajectories satisfying (19); we call this the primal behavior. The dual behavior is the set $\mathfrak{B}^{\prime}$ of all infinitely-differentiable trajectories $\operatorname{col}\left(u^{\prime}, y^{\prime}\right)$ such that

$$
\int_{-\infty}^{+\infty} u^{*} y^{\prime}+y^{*} u^{\prime} d t=0
$$

for all $\operatorname{col}(u, y) \in \mathfrak{B}$ of compact support. It can be proved (see [29]) that $\mathfrak{B}^{\prime}$ has the kernel representation

$$
D\left(-\frac{d}{d t}\right)^{\top} y^{\prime}=N\left(-\frac{d}{d t}\right)^{\top} u^{\prime}
$$

and the image representation

$$
\operatorname{col}\left(u^{\prime}, y^{\prime}\right)=\left[\begin{array}{l}
P\left(-\frac{d}{d t}\right)^{\top} \\
Q\left(-\frac{d}{d t}\right)^{\top}
\end{array}\right] \ell^{\prime} .
$$

Now denote $R:=\left[\begin{array}{ll}P & -Q\end{array}\right]$ and $M:=\left[\begin{array}{c}D \\ N\end{array}\right] ;$ it follows from (20) and (23) that $\mathfrak{B}=\operatorname{im} M\left(\frac{d}{d t}\right)$ and $\mathfrak{B}^{\prime}=$ $\operatorname{im} R\left(-\frac{d}{d t}\right)^{\top}$. Moreover, since $R M=0$, it follows (see 
Prop. 10.1 of [29]) that there exists a two-variable polynomial matrix $\Psi \in \mathbb{R}^{p \times m}[\zeta, \eta]$ such that $(\zeta+\eta) \Psi(\zeta, \eta)=$ $R(-\zeta) M(\eta)$. It also follows from Prop. 10.1 of [29] that $\Psi(\zeta, \eta)$ can be factored as $\Psi(\zeta, \eta)=Z(\zeta)^{\top} X(\eta)$, where $Z \in \mathbb{R}^{n \times p}[\xi]$ and $X \in \mathbb{R}^{n \times m}[\xi]$ have the property that $x:=X\left(\frac{d}{d t}\right) \ell$ and $x^{\prime}:=Z\left(\frac{d}{d t}\right) \ell^{\prime}$ are the state trajectories corresponding to $\operatorname{col}(u, y)$ and $\operatorname{col}\left(u^{\prime}, y^{\prime}\right)$ defined by (20) and (23), respectively (see [9], [24], [26] for a thorough introduction to such polynomial-differential operators). Consequently the integral relation (21) between primal and dual trajectories also admits a differential version

$$
u^{*} y^{\prime}+y^{*} u^{\prime}=\frac{d}{d t}\left(x^{*} x\right)
$$

which coincides with that obtained in Prop. 1 using statespace representations.

It can be shown (see Prop.s 2.4, 2.6 and 2.7 of [18], respectively) that results analogous to those illustrated in (9), Prop. 3 and Prop. 2 hold also in the higher-order case.

\section{CONCLUSIONS}

Several results in the Loewner framework for interpolation have been interpreted in the light of the concept of duality of dynamical systems. We have shown how the duality point of view allows novel insights of a more fundamental nature on some crucial results in the Loewner framework (e.g. the correspondence between state trajectories and factorizations in equation (24)). Moreover, known results in the Loewner framework have been shown to follow in a straightforward way from the relation between primal- and dual trajectories (e.g. the rank result of Prop. 2, the Sylvester equation in Prop. 3).

Current research directions include the formulation of recursive interpolation algorithms in the BDF framework, and the extension of the results presented here to parametric modelling and modelling of vector-exponential multidimensional trajectories.

\section{REFERENCES}

[1] B.D.O. Anderson and A.C. Antoulas, Rational interpolation and state variable realizations, Lin. Alg. Appl. 137/138:479-509 (1990).

[2] A.C. Antoulas, Approximation of large-scale dynamical systems, Advances in Design and Control, DC 06, SIAM, Philadelphia, 2nd edition (2008).

[3] A.C. Antoulas, On the construction of passive models from frequency response data, Automatisierungstechnik, AT-56, 447-452 (2008).

[4] A.C. Antoulas and B.D.O. Anderson, On the scalar rational interpolation problem, IMA J. of Mathematical Control and Information, 3: 61-88 (1986).

[5] A.C. Antoulas, S. Lefteriu and A.C. Ionita, A tutorial introduction to the Loewner framework for model reduction, in P. Benner, A. Cohen, M. Ohlberger and K. Willcox, Eds., Model reduction and approximation for complex systems, Birkhäuser, ISNM series (2015).

[6] A.C. Antoulas and J.C. Willems, A behavioral approach to linear exact modeling, IEEE Trans. Aut. Contr., AC-38: 1776-1802 (1993).

[7] D. Cobb, Controllability, observability, and duality in singular systems, IEEE Trans. Aut. Contr., AC-29(12):1076-1082 (1984).

[8] B. De Moor M. Moonen, L. Vandenberghe, and J. Vandewalle. Onand off-line identification of linear state-space models. Int. J. Contr., 49(1):219-232 (1989).

[9] P. A. Fuhrmann, P. Rapisarda, and Y. Yamamoto, On the state of behaviors, Lin. Alg. Appl., 424:2-3, 570-614 (2007).
[10] A. Ilchmann and V. Mehrmann, A behavioral approach to timevarying linear systems. Part 1: General theory. SIAM J. Contr. Opt., 44(5): 1725-1747 (2005).

[11] A. Ilchmann and V. Mehrmann, A behavioral approach to timevarying linear systems. Part 2: Descriptor systems. SIAM J. Contr. Opt., 44(5):1748-1765 (2005).

[12] O. Kaneko and P. Rapisarda, Recursive exact $H_{\infty}$-identification from impulse-response measurements, Syst. Contr. Lett., 49: 323-334 (2003).

[13] O. Kaneko and P. Rapisarda, On the Takagi interpolation problem,Lin. Alg. Appl., 425:2-3: 453-470 (2007).

[14] S. Lefteriu and A.C. Antoulas, A New Approach to Modeling Multiport Systems from Frequency-Domain Data, IEEE Trans. CAD, 29:14-27 (2010).

[15] S. Lefteriu and A.C. Antoulas, Topics in Model Order Reduction with Applications to Circuit Simulation, In: P. Benner, M. Hinze, E.J.W. ter Mater, Model Reduction for Circuit Simulation, Lecture Notes in Electrical Engineering, 74, 2011.

[16] A.J. Mayo, A.C. Antoulas, A behavioural approach to positive real interpolation, J. Math. Comp. Mod. Dyn. Syst., 8:445-455 (2002).

[17] A.J. Mayo and A.C. Antoulas, A framework for the solution of the generalized realization problem, 425:634-662, (2007).

[18] P. Rapisarda and A.C. Antoulas, Bilinear differential forms and the Loewner framework for rational interpolation, in Mathematical Control Theory II: Behavioral Systems and Robust Control, M.N. Belur, M.K. Çamlibel, P. Rapisarda and J.M.A. Scherpen, Eds., Lecture Notes in Control and Information Sciences, vol. 462, Springer-Verlag, 2015.

[19] P. Rapisarda and S. Rao, Realization of lossless systems via constant matrix factorizations, IEEE Trans. Aut. Contr., 58:2632-2636 (2013).

[20] P. Rapisarda and A.J. van der Schaft, Canonical realizations by factorization of constant matrices, Syst. Contr. Lett., 61(8): 827-833 (2012).

[21] P. Rapisarda and A.J. van der Schaft, Identification and data-driven reduced-order modeling for linear conservative port- and self-adjoint Hamiltonian systems, Proc. 52nd IEEE CDC, Firenze, Italy, 145-150 (2013).

[22] P. Rapisarda and H.L. Trentelman, Linear Hamiltonian behaviors and bilinear differential forms, SIAM J. Contr. Opt., 43:3, 769-791 (2004).

[23] P. Rapisarda and H.L. Trentelman, Identification and data-driven model reduction of state-space representations of lossless and dissipative systems from noise-free data, Automatica, 47:1721-1728 (2011).

[24] P. Rapisarda and J.C. Willems, State maps for linear systems, SIAM J. Contr. Opt., 35:3, 1053-1091 (1997).

[25] P. Rapisarda and J.C. Willems, The subspace Nevanlinna interpolation problem and the most powerful unfalsified model, Syst. Contr. Lett., 32: 291-300 (1997).

[26] A.J. van der Schaft and P. Rapisarda, State maps from integration by parts, SIAM J. Contr. Opt., 49: 2415-2439 (2011).

[27] J.C. Willems, From time series to linear system - Part II. Exact modelling, Automatica, 22: 675-694 (1986).

[28] J.C. Willems, Paradigms and puzzles in the theory of dynamical systems, IEEE Trans. Aut. Contr., 36: 259-294 (1991).

[29] Willems, J.C. and H.L. Trentelman, On quadratic differential forms, SIAM J. Control Opt., 36:1703-1749 (1998). 